Aljazi et al.

\title{
Histone H3K36me2-specific methyltransferase ASH1L is required for the MLL-AF9-induced leukemogenesis
}

\author{
Mohammad B. Aljazi ${ }^{1}$, Yuen Gao ${ }^{1}$, Yan Wu ${ }^{1}$, George I Mias ${ }^{1,2}, \operatorname{Jin}_{\mathrm{He}^{1 *}}$
}

${ }^{1}$ Department of Biochemistry \& Molecular Biology, College of Nature Sciences, Michigan State University, East Lansing, MI 48824

${ }^{2}$ Institute for Quantitative Health Science and Engineering, Michigan State University, East Lansing, MI 48824

* Correspondence: Jin He

Email: hejin1@msu.edu 
Aljazi et al.

\section{Abstract}

ASH1L and MLL1 are two histone methyltransferases that facilitate transcriptional activation during normal development. However, the roles of ASH1L and its enzymatic activity in the development of MLL-rearranged leukemias are not fully elucidated in the AshlL gene knockout animal models. In this study, we used an AshlL conditional knockout mouse model to show that loss of ASH1L in hematopoietic progenitor cells impaired the initiation of MLL-AF9-induced leukemic transformation in vitro. Furthermore, genetic deletion of ASH1L in the MLL-AF9transformed cells impaired the maintenance of leukemic cells in vitro and largely blocked the leukemia progression in vivo. Importantly, the loss of ASH1L function in the Ash1L-deleted cells could be rescued by wild-type but not the catalytic-dead mutant ASH1L, suggesting the enzymatic activity of ASH1L was required for its function in promoting MLL-AF9-induced leukemic transformation. At the molecular level, ASH1L enhanced the MLL-AF9 target gene expression by directly binding to the gene promoters and modifying the local histone H3K36me2 levels. Thus, our study revealed the critical functions of ASH1L in promoting the MLL-AF9-induced leukemogenesis, which provides a molecular basis for targeting ASH1L and its enzymatic activity to treat MLL-arranged leukemias.

\section{Key words}

MLL-rearranged leukemia, MLL1, ASH1L, histone modification, H3K36me2, leukemogenesis

\section{Introduction}

The MLL rearrangement (MLLr) caused by 11q23 chromosomal translocations creates a variety MLL fusion proteins that drive the acute lymphoblastic and myeloid leukemia development, which accounts for approximate 5-10\% acute leukemias in human patients[1-5]. Despite recent progression in the development of chemotherapies against leukemias, the overall prognosis for the MLLr leukemias remains poor[6, 7].

MLL1 protein is a histone lysine methyltransferase (KMTase) that contains a SET ( $\underline{S}$ (var)3-9, Enhancer-of-zeste and Trithorax) domain to catalyze trimethylation of histone H3 lysine 4 (H3K4me3)[8] . Functionally, MLL1 belongs to the Trithorax-group (TrxG) proteins that antagonize the Polycomb-group (PcG)-mediated gene silencing and facilitate transcriptional activation[9]. In 11q23 chromosomal translocations, the $N$-terminal portion of MLL1 is fused with a variety of fusion partners to generate different oncogenic MLL fusion proteins that function as 
Aljazi et al.

disease drivers leading to leukemia development[10-12]. Previous studies have revealed that the $N$ terminal portion of MLL fusion proteins interacts with MENIN and LEDGF ( $\underline{\text { ens }} \underline{\text { Epithelium- }}$ $\underline{D}$ erived $\underline{G}$ rowth $\underline{F}$ actor), which is critical for the recruitment of MLL fusion proteins to chromatin, whereas the $C$-terminal fusion partners interact with various trans-activators to induce transcriptional activation[13-17]. However, since the MLL fusion proteins lack the intrinsic histone H3K4 methyltransferase activity due to loss of SET domain located in the $C$-terminal portion of MLL1[10], it is unclear whether other histone modifications are required for the MLL fusion proteins-induced gene expression and leukemogenesis.

Recently, another member of TrxG proteins, ASH1L ( $\underline{A}$ bsent, $\underline{S}$ mall, or $\underline{H}$ omeotic- $\underline{L}$ ike $\underline{1}$ ), was found to play important roles in normal hematopoiesis and leukemogenesis[8, 18, 19]. Biochemically, ASH1L is a histone KMTase that mediates dimethylation of histone H3 lysine 36 (H3K36me2)[20]. Similar to MLL1, ASH1L facilitates gene expression through antagonizing PcGmediated gene silencing[8]. Previous studies have shown that ASH1L and MLL1 co-occupies the same transcriptional regulatory regions, and loss of either ASH1L or MLL1 reduces the expression of common genes[21-23], suggesting ASH1L and MLL1 function synergistically to activate gene expression during normal development. However, the significance of ASH1L and its-mediated histone H3K36me2 in the MLLr-associated leukemogenesis has not been addressed in the AshlL gene knockout animal models.

In this study, we used an Ash1L conditional knockout mouse model to show that loss of ASH1L in hematopoietic progenitor cells (HPCs) impaired the initiation of MLL-AF9-induced leukemic transformation in vitro. Furthermore, genetic deletion of ASH1L in the MLL-AF9-transformed cells impaired the maintenance of leukemic cells in vitro and largely blocked the leukemia progression in vivo. Importantly, the loss of ASH1L function in the Ash1L-deleted cells could be rescued by ectopic expression of wild-type but not the catalytic-dead mutant ASH1L, suggesting the enzymatic activity of ASH1L was required for its function in promoting MLL-AF9-induced leukemic transformation. At the molecular level, ASH1L activated the MLL-AF9 target gene expression by directly binding to the gene promoters and modifying the local histone H3K36me2 levels. Thus, our study revealed the critical functions of ASH1L in MLL-AF9-induced leukemogenesis and raised the possibility that ASH1L might serve as a potential therapeutic target for the treatment of MLLr leukemias.

\section{Materials and Methods:}


Aljazi et al.

The Ash1L conditional knockout mice were generated as previously reported[24]. To generate inducible Ash1L deletion, mice were crossed with Rosa26-CreER ${ }^{\mathrm{T} 2}$ mice that were obtained from The Jackson Laboratory. All mice for this study were backcrossed to C57BL/6 mice for at least five generations to reach pure genetic background prior to conducting experiments. All mouse experiments were performed with the approval of the Michigan State University Institutional Animal Care \& Use Committee.

\section{Hematopoietic progenitor isolation, and culture}

Hematopoietic progenitor cells were isolated from femurs of 4- to 6-week C57BL/6 mice. The red blood cells in the bone marrows were lysed by ammonium chloride solution (Stem Cell Technologies 07800) and filtered with a 70- $\mu \mathrm{m}$ nylon filter. The c-kit ${ }^{+}$HPCs were isolated using cKit antibody-conjugated IMag (BD Biosciences) beads. Cultures of transformed HPCs cells were maintained in RMPI media supplemented with 10\% FBS, 1\% MEM non-essential amino acids, $1 \%$ Glutamax, 10 ng/mL, and 2-mercaptoethanol. To induce Cre-mediated recombination in vitro, 4hydroxy-tamoxifen (Sigma-Aldrich) was resuspended in DMSO and supplemented into the culture medium with concentration of $250 \mathrm{nM}$.

\section{Retroviral and lentiviral vector production and transduction}

The pMIG-FLAG-MLL-AF9 retroviral vectors as obtained from Addgene (Plasmid \#71443). Retroviral vectors were generated by co-transfection of retroviral vectors with pGag-pol, pVSVG 293T cells using CalPhos mammalian transfection kit (TaKaRa). After 48hrs post transfection, viral supernatant was harvested, filtered through a $0.45 \mu \mathrm{m}$ membrane, and concentrated by ultracentrifugation. The lentiviral system was obtained from the National Institutes of Health AIDS Research and Reference Reagent Program. To generate GFP expression vectors, the GFP cDNA was PCR amplified, fused with P2A and puromycin resistant cassette and cloned into the SpeI/EcoRI sites under the EF1 $\alpha$ promoter. To generate lentiviral viruses, the transducing vectors pTY, pHP and pHEF1 $\alpha-V S V G$ were co-transfected into HEK293T cells. The supernatant was collected at 24, 36 and 48 hours after transfection, filtered through a $0.45 \mu \mathrm{m}$ membrane and concentrated by ultracentrifugation. Retroviral and lentiviral transduction of HPCs was performed by spin inoculation for 1 hour at 800g, in RMPI media supplemented with 10\% FBS, 1x MEM nonessential amino acids (Life Technologies), 1x Glutamax (Life Technologies), 1x sodium pyruvate (Life Technologies), 50 ng/mL mSCF (PeproTech), 10 ng/mL mIL-6 (PeproTech), and 10 ng/mL mIL-3 (PeproTech). 
Aljazi et al.

\section{Serial methylcellulose replating assay and leukemia transplantation}

The colony formation assays were conducted by plating 500 cells into methylcellulose media consisting of Iscove MDM (Life Technologies) supplemented with FBS, BSA, insulin-transferrin (Life Technologies), 2-mercaptoethanol, mSCF, mIL-3, mIL-6, and 10 ng/mL GM-CSF (PeproTech). After 7-10 days, the colony numbers were counted under a microscope. The colonies were picked up, and cells were pooled and replated onto secondary methylcellulose plates. Three rounds of replating were performed for each experiment. For leukemia transplantation, recipient C57BL/6 mice were subjected to total body irradiation at a dose of $11 \mathrm{~Gy}$ with the use of a X-RAD 320 biological irradiator. Donor cells $\left(5 \times 10^{5}\right)$ and radiation protector cells $\left(5 \times 10^{5}\right)$ isolated from $\mathrm{BM}$ were mixed in $1 \times \mathrm{PBS}$ and transplanted into the recipient mice through retro-orbital injection. The mice were fed with water supplemented with trimethoprim/sulfamethoxazole for 4 weeks after transplantation.

\section{FACS analysis}

For FACS analysis, cells were stained with antibodies in staining buffer (1× PBS, 2\% FBS) and incubated at $4^{\circ} \mathrm{C}$ for 30 minutes. The samples were washed once with staining buffer before subjected to FACS analysis with the use of a BD LSRII. The antibodies used in this study include anti-Mac-1(eBioscience), anti-Gr-1(eBioscience), anti-c-kit (eBioscience).

\section{Western Blot analysis}

Total proteins were extracted by RIPA buffer and separated by electrophoresis by 8-10\% PAGE gel. The protein was transferred to the nitrocellulose membrane and blotted with primary antibodies. The antibodies used for Western Blot and IP-Western Blot analyses included: rabbit anti-Flag (1:1000, cell signaling) and IRDye 680 donkey anti-rabbit second antibody (1: 10000, Li-Cor). The images were developed by Odyssey Li-Cor Imager (Li-Cor).

\section{Quantitative RT-PCR and ChIP-qPCR assays}

RNA was extracted and purified from cells with the use of Qiashredder (QIAGEN) and RNeasy (QIAGEN) spin columns. Total RNA $(1 \mu \mathrm{g})$ was subjected to reverse transcription using Iscript reverse transcription supermix (Bio-Rad). cDNA levels were assayed by real-time PCR using iTaq universal SYBR green supermix (Bio-Rad) and detected by CFX386 Touch Real-Time PCR detection system (Bio-Rad). Primer sequences for qPCR are listed in Supplementary Table 5 The expression of individual genes is normalized to expression level of Gapdh. ChIP assays that used rabbit anti-ASH1L antibody (in house), rabbit anti-H3K36me2 antibody (Abcam), rabbit anti-MLL- 
Aljazi et al.

AF9-Flag (Cell signaling) were carried out according to the previously reported protocol with the following modifications: 2 ug antibodies were used in the immunoprecipitation, and chromatinbound beads were washed 3 times each with TSEI, TSEII, and TESIII followed by 2 washes in 10mM Tris, pH 7.5, 1mM EDTA. Histone modification ChIPs were carried out as previously reported. DNA that underwent ChIP was analyzed by quantitative PCR (qPCR), and data are presented as the percentage of input as determined with CFX manager 3.1 software. Primers for qPCR and ChIP assays are listed in supplementary Tables S3, respectively.

\section{RNA-seq sample preparation for HiSeq4000 sequencing}

RNA was extracted and purified from cells using QI shredder (Qiagen) and RNeasy (Qiagen) spin columns. Total RNA ( $1 \mu \mathrm{g}$ ) was used to generate RNA-seq library using NEBNext Ultra Directional RNA library Prep Kit for Illumina (New England BioLabs, Inc) according to the manufacturer's instructions. Adapter-ligated cDNA was amplified by PCR and followed by size selection using agarose gel electrophoresis. The DNA was purified using Qiaquick gel extraction kit (Qiagen) and quantified both with an Agilent Bioanalyzer and Invitrogen Qubit. The libraries were diluted to a working concentration of $10 \mathrm{nM}$ prior to sequencing. Sequencing on an Illumina HiSeq4000 instrument was carried out by the Genomics Core Facility at Michigan State University.

\section{RNA-seq data analysis}

RNA-Seq data analysis was performed essentially as described previously. All sequencing reads were mapped mm9 of the mouse genome using Tophat2[25]. The mapped reads were normalized to reads as Reads Per Kilobase of transcript per Million mapped reads (RPKM). The differential gene expression was calculated by Cuffdiff program and the statistic cutoff for identification of differential gene expression is $p<0.01$ and 1.5 -fold RPKM change between samples[26]. The heatmap and plot of gene expression were generated using plotHeatmap and plotProfile in the deepTools program[27]. The differential expressed gene lists were input into the David Functional Annotation Bioinformatics Microarray Analysis for the GO enrichment analyses (https://david.ncifcrf.gov/).

\section{Statistical analysis}

All statistical analyses were performed using GraphPad Prism 8 (GraphPad Software). Parametric data were analyzed by a two-tailed $t$ test or two-way ANOVA test for comparisons of multiple samples. $P$ values $<0.05$ were considered statistically significant. Data are presented as mean \pm SEM. 
Aljazi et al.

Results

\section{ASH1L is required for the initiation of MLL-AF9-induced leukemic transformation in vitro}

To examine the function of ASH1L in MLLr-induced leukemogenesis, we generated an Ash1L conditional knockout $(A \operatorname{sh} 1 L-\mathrm{cKO})$ mouse line in which two LoxP elements inserted into the exon 4 flanking regions[24]. A CRE recombinase-mediated deletion of exon 4 resulted in altered splicing of mRNA that created a premature stop codon before the sequences encoding the first functional AWS ( $\underline{A}$ ssociated $\underline{W}$ ith $\underline{S} E T)$ domain (Fig. S1a, S1b). The AshlL-cKO mice were further crossed with the Rosa26-CreER ${ }^{\mathrm{T} 2}$ mice to generate a tamoxifen-inducible Ash1L knockout line $\left(A s h 1 L^{2 \mathrm{f} / 2 \mathrm{f}} ;\right.$ Rosa26-CreER $\left.{ }^{\mathrm{T} 2}\right)$, which allowed us to study the function of ASH1L in leukemogenesis in vitro and in vivo.

Using this $A s h 1 L$-cKO mouse model, we set out to investigate the role of ASH1L in the initiation of MLL-AF9-induced leukemic transformation. To this end, we isolated the bone marrow cells from wild-type $\left(A \operatorname{sh} 1 L^{+/+} ; C r e-E R^{T 2}\right)$ and $A s h 1 L-c K O\left(A s h 1 L^{2 f / 2 f} ; C r e-E R^{T 2}\right)$ mice, respectively. The c-kit ${ }^{+}$ HPCs were further enriched by the c-kit antibody-conjugated magnetic beads (Fig. S1c). The HPCs were cultured in the HPC medium supplemented with murine IL-3, IL-6, and SCF for three days, and followed by transduction of retroviral vectors expressing a MLL1-AF9 fusion gene or control empty viruses (EV). After transduction, the cells were cultured in the suspension medium with 4hydroxytamoxifen (4-OHT) for five days to induce Ash $1 L$ gene deletion in the AshlL-cKO HPCs (Fig. 1a). The quantitative RT-PCR (qRT-PCR) analysis showed that the $A s h 1 L$ expression reduced to less than 5\% at the mRNA level in the Ash1L-deleted cells (Fig. 1b). To investigate the effect of Ash1L loss on the initiation of MLL-AF9-induced leukemic transformation in vitro, we performed the serial colony replating assays by plating the cells on the semi-solid methylcellulose medium to examine the leukemic transformation. The results showed that although the cells transduced with MLL-AF9 or empty vectors had comparable colony numbers in the first round of plating, the cells transduced with control empty vectors did not form colonies in the following rounds of replating. In contrast, both wild-type and $A s h 1 L$-cKO HPCs transduced with $M L L-A F 9$ retroviruses formed colonies in all three rounds of plating, indicating the successful leukemic transformation by the MLL-AF9 transgene in vitro. Notably, compared to the MLL-AF9-transduced wild-type cells, the AshlL-deleted cells had reduced colony numbers in the second and third rounds of plating, suggesting that loss of Ash1L in HPCs compromised the MLL-AF9-induced leukemic transformation (Fig. 1c, d). Further qRT-PCR analyses showed that the MLL-transformed wild-type and $A s h 1 L$-cKO cells had the comparable expression levels of $M L L-A F 9$ transgene, suggesting that 
Aljazi et al.

the difference in colony numbers was not due to a disparity in $M L L-A F 9$ transgene gene expression (Fig. S1d).

\section{ASH1L is required for the maintenance of MLL-AF9-induced leukemic cells in vitro}

Next, we set out to examine whether Ash $1 L$ was required for the maintenance of MLL-AF9 transformed cells. To this end, we transduced both wild-type and Ash1L-cKO HPCs with $M L L-A F 9$ retroviruses and plated the transduced cells onto the methylcellulose medium. After three rounds of replating, the transformed colonies were manually picked and cultured in the suspension medium supplemented with 4-OHT for 5 days to induce deletion of AshlL in the AshlL-cKO cells. The cells were further maintained in suspension culture without 4-OHT for 5 days before plated onto the methylcellulose to examine the colony forming capacity (Fig. 2a). The results showed that compared to the wild-type MLL-AF9-transformed cells, the Ash1L-deleted cells had marked reduced colony formation (Fig. 2b, c), suggesting that ASH1L was required for the maintenance of MLL-AF9 transformed cells in vitro.

To examine the cellular responses to the AshlL depletion, we performed the FACS analysis to examine the cell death in response to the loss of Ash1L in the MLL-AF9-transformed cells. The results showed that compared to the wild-type cells, the AshlL-deleted cells had increased populations of both early apoptotic cells (Annexin V+/DAPI-) and late dead cells (Annexin V+/DAPI+) (Fig. 2d), suggesting that the loss of Ash1L induced cell death of MLL-AF9transformed cells. Moreover, FACS analyses showed that compared to the wild-type transformed cells, the $A s h 1 L$-deleted cells had increased expression of myeloid differentiation surface markers CD11b and GR-1 (Fig. 2e). Morphologically, the wild-type transformed cells displayed leukoblastlike morphology with enlarged dark stained nuclei, while the $A s h 1 L$-deleted cells had light-stained and segmented nuclei, a feature indicating the differentiation towards matured myeloid cells (Fig. 2f). Taken together, these results suggested that ASH1L was required for the maintenance of MLLAF9-transformed cells through suppressing cell death and differentiation.

\section{ASH1L is required for the MLL-AF9-induced leukemia development in vivo}

To determine whether ASH1L was required for the MLL-AF9-induced leukemogenesis in vivo, we performed leukemia transplantation assays and monitor the leukemia development in the recipient mice. To this end, the wild-type and Ash1L-deleted MLL-AF9-transformed cells were labeled with GFP by transduction with lentiviral-GFP vectors, mixed with normal protective bone marrow cells, and transplanted into the total-body-irradiated (TBI) syngeneic recipient mice (Fig. 3a). Four weeks 
Aljazi et al.

after transplantation, FACS analysis showed that the mice transplanted with wild-type leukemic cells had higher GFP+ leukemic cell populations in the peripheral blood compared to the mice received with Ash1L-KO leukemic cells (Fig. 3b), which was consistent with the higher leukemic cell numbers in the peripheral blood smears and splenomegaly found in the mice transplanted with wild-type leukemic cells (Fig. 3c, d). All mice transplanted with wild-type leukemic cells died within 3 months after transplantation, and the median survival time was around 9 weeks. In contrast, the median survival time for the mice transplanted with Ash $1 L$-deleted cells was around 12.5 weeks and the overall survival time was 4 weeks longer than the mice transplanted with wild-type leukemic cells (Fig. 3e). These results suggested that ASH1L in the MLL-AF9-transformed leukemic cells was required for the development and progression of leukemia in vivo.

\section{The enzymatic activity of ASH1L is required for its function in promoting MLL-AF9-induced leukemic transformation}

Next, we set out to determine whether the histone methyltransferase activity of ASH1L was required for its function in promoting MLL-AF9-induced leukemic transformation. To this end, the AshlLcKO HPCs were infected with retroviruses expressing $M L L-A F 9$ transgene, followed by transduced with lentiviral vectors expressing either wild-type ASH1L or catalytic-dead mutant ASH1L(H2214A)[21]. The transformed cells were treated with 4-OHT to induce deletion of endogenous AshlL gene (Fig. 4a). WB analysis showed that both wild-type and mutant exogenous ASH1L had a similar expression level (Fig. 4b). The cells were further plated onto the methylcellulose medium to examine the colony formation (Fig. 4a). The results showed that compared to the wild-type ASH1L expressed cells, the cells with ectopic expression of catalyticdead mutant ASH1L had reduced colony formation (Fig 4c). Similar to the Ash1L-deleted cells, the Ash1L-deleted cells rescued with mutant ASH1L had increased cell death and upregulated expression of myeloid differentiation markers of CD11b and GR-1 (Fig. 4e, f). These results suggested that ASH1L histone methyltransferase activity was required for its function in promoting MLL-AF9-induced leukemogenesis by inhibiting cell death and blocking myeloid differentiation.

\section{ASH1L facilitates the MLL-AF9-induced leukemogenic gene expression}

To examine the molecular mechanisms underlying the function of ASH1L in promoting MLL-AF9induced leukemogenesis, we performed the RNA-seq analysis to examine the transcriptome changes in normal HPCs, wild-type and Ash1L-deleted MLL-AF9-tranformed cells. The results showed that compared to normal HPCs, the MLL-AF9-transformed cells had 1,021 upregulated and 1,228 downregulated genes (cutoff: fold changes $>1.5$, FDR $<0.05$ ), respectively (Fig. 5a). The 
Aljazi et al.

gene ontology (GO) enrichment analysis showed that both upregulated and downregulated genes were involved in immune processes and inflammatory responses (cutoff: FDR < 0.05 ) (Tables S1 and S2), suggesting that MLL-AF9 fusion proteins disrupted the normal differentiation and misregulated the normal function of myeloid cells. Notably, multiple genes, such as Hoxa5, Hoxa7, Hoxa9, Hoxal0 and MeisI that were known to mediate the MLL-AF9-induced leukemogenesis, were highly expressed in the MLL-AF9-transformed cells (Fig. 5b). Further RNA-seq analysis showed that compared to MLL-AF9-transformed wild-type cells, the Ash1L-deleted cells had 372 upregulated gene and 472 downregulated genes (cutoff: fold changes > 1.5, FDR < 0.05), respectively (Fig. 5c). Cross-examining these two data sets revealed that 105 genes, including Hoxa5, Hoxa7, Hoxa9, Hoxa10, and MeisI that were highly expressed in the wild-type MLL-AF9transformed cells, were downregulated in the AshlL-deleted cells (Fig. 5d, e). Overall, these results suggested that ASH1L promoted the MLL-AF9-induced leukemogenesis by facilitating the MLLAF9-induced leukemic gene expression.

\section{ASH1L binds and mediates the histone H3K36me2 modification at Hoxa9 and Hoxa10 gene promoters}

To determine whether ASH1L directly regulated the expression of MLL-AF9 target genes, we performed chromatin immunoprecipitation (ChIP) coupled with quantitative PCR (ChIP-qPCR) assays to examine the ASH1L occupancy, MLL-AF9 occupancy, and histone H3K36me2 modification at the gene promoters, transcriptional starting sites (TSS), transcriptional ending sites (TES) of Hoxa9 and Hoxa10, two MLL-AF9 target genes that were shown to be activated in the wild-type transformed cells and have reduced expression in the AshlL-deleted cells (Fig. 5b, e). The results showed that both ASH1L occupancy and histone H3K36me2 were enriched at the Hoxa9 and HoxalO promoters compared to that on the TES and the long terminal repeat (LTR) of endogenous retroviruses. Furthermore, compared to wild-type MLL-AF9-transformed cells, both ASH1L occupancy and histone H3K36me2 modification were reduced at the gene promoters in the Ash1L-deleted cells, suggesting that ASH1L bound to the Hoxa9 and HoxalO gene promoters directly and mediated local histone H3K36me2 modification. However, the MLL-AF9 occupancy at both gene promoters did not show significant difference between wild-type and $A s h 1 L$-deleted MLL-AF9-transformed cells, suggesting the ASH1L-mediated histone H3K36me2 did not to affect the binding of MLL-AF9 fusion protein to the gene promoters. 
Aljazi et al.

\section{Discussion}

Chromosomal 11q23 translocations generate various MLL fusion proteins that contain the $N$ terminal portion of MLL1 and different fusion partners including AF9[28, 29]. Previous studies have demonstrated that the $N$-terminal MLL1 is critical for the recruitment of MLL fusion proteins to chromatin through its $\mathrm{CxxC}$-zinc finger ( $\mathrm{CxxC}-\mathrm{zf})$ domain as well as its interacting proteins MENIN and LEDGF, while the $C$-terminal fusion partners interact with multiple trans-activators to induce transcriptional activation[16]. Since the MLL fusion proteins lose the MLL1 $C$-terminal SET domain and its-associated histone H3K4 methyltransferase activity, it is unclear whether other histone KMTase-mediated histone modifications are required for the MLL fusion proteins to activate leukemogenic gene expression and induce leukemia development.

ASH1L is another member of TrxG proteins that facilitate transcriptional activation[8]. Biochemically, ASH1L is a histone KMT mediating histone H3K36me2 modification[20]. Recent studies reported that ASH1L and MLL1 co-occupied the same gene promoters to activate gene expression, suggesting ASH1L and MLL function synergistically in activating gene expression in normal development and leukemogenesis[19, 21-23]. However, the functional roles of ASH1L and its-mediated histone H3K36me 2 in the MLLr leukemia development have not been addressed using Ash1L gene knockout animal models.

In this study, we used an Ash1L conditional knockout mouse model to show that ASH1L and its histone methyltransferase activity are required for the MLL-AF9-induced leukemogenesis. First, genetic deletion of ASH1L in normal HPCs largely impairs the MLL-AF9-induced colony formation in serial methylcellulose replating assays (Fig. 1), suggesting ASH1L is required for the initiation of MLL-AF9-induced leukemic transformation. Second, loss of ASH1L in the MLL-AF9transformed cells largely impaired the colony formation in vitro and delayed the leukemia development in the recipient mice transplanted with leukemic cells (Figs. 2 and 3), suggesting ASH1L is required for the maintenance of MLL-AF9-transformed cells in vitro and leukemia progression in vivo. Importantly, the impaired ASH1L's function in the Ash $1 L-\mathrm{KO}$ cells could be rescued by the wild-type but not the catalytic-dead mutant ASH1L (Fig. 4), suggesting that the histone methyltransferase activity is required for its function in promoting MLL-AF9-induced leukemogenic transformation, which is consistent with a recent study showing that the SET domain is required for the MLL-AF9-induced leukemic transformation[30].

At the cellular level, we observed that the loss of ASH1L in MLL-AF9-transformed cells induced cell death and myeloid differentiation, which could be rescued by the wild-type but not the catalytic-dead mutant ASH1L (Figs. 2 and 4), suggesting that ASH1L promotes MLL-AF9-induced leukemic transformation though inhibit cell apoptosis and block cell differentiation. These results 
Aljazi et al.

are consistent with the molecular findings that ASH1L is required for the full activation of MLLAF9 target genes including Hoxa gene cluster and MeisI (Fig. 5), which were known to play important roles in leukemogenesis through inhibiting cell death and blocking normal cell differentiation[31-33]. Finally, the ChIP assays showed that both ASH1L occupancy and histone H3K36me2 modification were enriched at the promoters of MLL-AF9 target genes Hoxa9 and Hoxa10 in the wild-type transformed cells (Fig. 6), indicating the ASH1L regulates the MLL-AF9 target genes through directly chromatin binding and its-mediated histone H3K36me2 modification.

Previous studies have shown that the PWWP domain of LEDGF is required for the recruitment of MLL fusion proteins through its binding to histone H3K36me2[13, 15, 16]. However, our ChIP analysis did not reveal the reduction of MLL-AF9 occupancy at the Hoxa9 and HoxalO promoters in the Ash1L-KO cells (Fig. 6f, g), suggesting the MLL-AF9 fusion protein could bind to its target regions though other recruiting mechanisms, such as through the $\mathrm{CxxC}$-zf domain binding to the unmethylated CpG-rich promoters[34], and the reduced H3K36me2 at gene promoters in the AshlL-KO cells might impair the Hoxa gene expression through mechanisms other than the recruitment of MLL-AF9 fusion protein.

In summary, our study reveals that the histone H3K36me2-specific methyltransferase ASH1L and its enzymatic activity play a critical role in promoting MLL-AF9-induced leukemogenesis, which provides an important molecular basis for targeting ASH1L and its enzymatic activity to treat MLL-r leukemias.

\section{Data availability statement}

The RNA-seq data presented in this study is being deposited to the Gene Expression Omnibus database. Other data have been disclosed in the article/Supplementary Material.

\section{Author contributions}

J.H. conceived the project. M.B.A., Y.G., Y.W. and J.H. performed the experiments. J.H. and G.I.M. performed the sequencing data analysis. J.H. interpreted the data and wrote the manuscript.

\section{Funding}

This work was supported by the National Institute Health NIH grant R01GM127431.

\section{Acknowledgements}

MSU genomics core facility processed the next-generation sequencing. 
Aljazi et al.

\section{Competing Interest Statement}

Authors declare no competing interests.

\section{References}

1. Berger, R., et al., Acute monocytic leukemia chromosome studies. Leuk Res, 1982. 6(1): p. 17-26.

2. Nakamura, T., et al., Genes on chromosomes 4, 9, and 19 involved in 11q23 abnormalities in acute leukemia share sequence homology and/or common motifs. Proc Natl Acad Sci U S A, 1993. 90(10): p. 4631-5.

3. De Braekeleer, M., et al., The MLL gene and translocations involving chromosomal band 11 q23 in acute leukemia. Anticancer Res, 2005. 25(3B): p. 1931-44.

4. Vermaelen, K., et al., Anomalies of the long arm of chromosome 11 in human myelo-and lymphoproliferative disorders. I. Acute nonlymphocytic leukemia. Cancer Genet Cytogenet, 1983. 10(1): p. 105-16.

5. Ayton, P.M. and M.L. Cleary, Molecular mechanisms of leukemogenesis mediated by MLL fusion proteins. Oncogene, 2001. 20(40): p. 5695-707.

6. Chessells, J.M., et al., Clinical features, cytogenetics and outcome in acute lymphoblastic and myeloid leukaemia of infancy: report from the MRC Childhood Leukaemia working party. Leukemia, 2002. 16(5): p. 776-84.

7. Hilden, J.M., et al., Analysis of prognostic factors of acute lymphoblastic leukemia in infants: report on CCG 1953 from the Children's Oncology Group. Blood, 2006. 108(2): p. 441-51.

8. Kingston, R.E. and J.W. Tamkun, Transcriptional regulation by trithorax-group proteins. Cold Spring Harb Perspect Biol, 2014. 6(10): p. a019349.

9. Schuettengruber, B., et al., Genome regulation by polycomb and trithorax proteins. Cell, 2007. 128(4): p. 735-45.

10. Slany, R.K., The molecular biology of mixed lineage leukemia. Haematologica, 2009. 94(7): p. 98493.

11. Winters, A.C. and K.M. Bernt, MLL-Rearranged Leukemias-An Update on Science and Clinical Approaches. Front Pediatr, 2017. 5: p. 4.

12. Chan, A.K.N. and C.W. Chen, Rewiring the Epigenetic Networks in MLL-Rearranged Leukemias: Epigenetic Dysregulation and Pharmacological Interventions. Front Cell Dev Biol, 2019. 7: p. 81.

13. Yokoyama, A., et al., The menin tumor suppressor protein is an essential oncogenic cofactor for MLL-associated leukemogenesis. Cell, 2005. 123(2): p. 207-18.

14. Shun, M.C., et al., Identification and characterization of PWWP domain residues critical for LEDGF/p75 chromatin binding and human immunodeficiency virus type 1 infectivity. J Virol, 2008. 82(23): p. 11555-67.

15. Yokoyama, A. and M.L. Cleary, Menin critically links MLL proteins with LEDGF on cancerassociated target genes. Cancer Cell, 2008. 14(1): p. 36-46.

16. Yokoyama, A., Transcriptional activation by MLL fusion proteins in leukemogenesis. Exp Hematol, 2017. 46: p. 21-30.

17. Okuda, H., et al., MLL fusion proteins link transcriptional coactivators to previously active CpG-rich promoters. Nucleic Acids Res, 2014. 42(7): p. 4241-56.

18. Jones, M., et al., Ash1l controls quiescence and self-renewal potential in hematopoietic stem cells. J Clin Invest, 2015. 125(5): p. 2007-20.

19. Zhu, L., et al., ASH1L Links Histone H3 Lysine 36 Dimethylation to MLL Leukemia. Cancer Discov, 2016. 6(7): p. 770-83.

20. Yuan, W., et al., H3K36 methylation antagonizes PRC2-mediated H3K27 methylation. J Biol Chem, 2011. 286(10): p. 7983-7989.

21. Miyazaki, H., et al., Ash1l methylates Lys36 of histone H3 independently of transcriptional elongation to counteract polycomb silencing. PLoS Genet, 2013. 9(11): p. e1003897. 
Aljazi et al.

22. Gregory, G.D., et al., Mammalian ASH1L is a histone methyltransferase that occupies the transcribed region of active genes. Mol Cell Biol, 2007. 27(24): p. 8466-79.

23. Tanaka, Y., et al., Dual function of histone H3 lysine 36 methyltransferase ASH1 in regulation of Hox gene expression. PLoS One, 2011. 6(11): p. e28171.

24. Gao, Y., et al., Loss of histone methyltransferase ASH1L in the developing mouse brain causes autistic-like behaviors. Commun Biol, 2021. 4(1): p. 756.

25. Kim, D., et al., TopHat2: accurate alignment of transcriptomes in the presence of insertions, deletions and gene fusions. Genome Biol, 2013. 14(4): p. R36.

26. Trapnell, C., et al., Differential analysis of gene regulation at transcript resolution with RNA-seq. Nat Biotechnol, 2013. 31(1): p. 46-53.

27. Ramirez, F., et al., deepTools: a flexible platform for exploring deep-sequencing data. Nucleic Acids Res, 2014. 42(Web Server issue): p. W187-91.

28. Krivtsov, A.V. and S.A. Armstrong, MLL translocations, histone modifications and leukaemia stemcell development. Nat Rev Cancer, 2007. 7(11): p. 823-33.

29. Dobson, C.L., et al., The mIl-AF9 gene fusion in mice controls myeloproliferation and specifies acute myeloid leukaemogenesis. EMBO J, 1999. 18(13): p. 3564-74.

30. Rogawski, D.S., et al., Discovery of first-in-class inhibitors of ASH1L histone methy/transferase with anti-leukemic activity. Nat Commun, 2021. 12(1): p. 2792.

31. Sitwala, K.V., M.N. Dandekar, and J.L. Hess, HOX proteins and leukemia. Int J Clin Exp Pathol, 2008. 1(6): p. 461-74.

32. Domsch, K., F. Papagiannouli, and I. Lohmann, The HOX-Apoptosis Regulatory Interplay in Development and Disease. Curr Top Dev Biol, 2015. 114: p. 121-58.

33. Magli, M.C., C. Largman, and H.J. Lawrence, Effects of HOX homeobox genes in blood cell differentiation. J Cell Physiol, 1997. 173(2): p. 168-77.

34. Ayton, P.M., E.H. Chen, and M.L. Cleary, Binding to nonmethylated CpG DNA is essential for target recognition, transactivation, and myeloid transformation by an MLL oncoprotein. Mol Cell Biol, 2004. 24(23): p. 10470-8. 
Aljazi et al.

Figures and figure legend

a

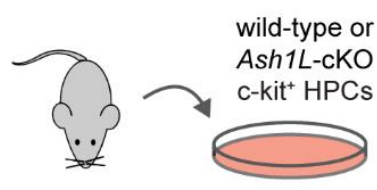

Methycellulose replating assay

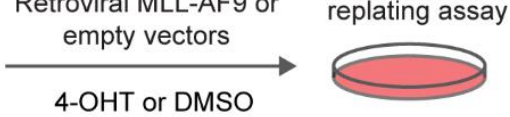

C

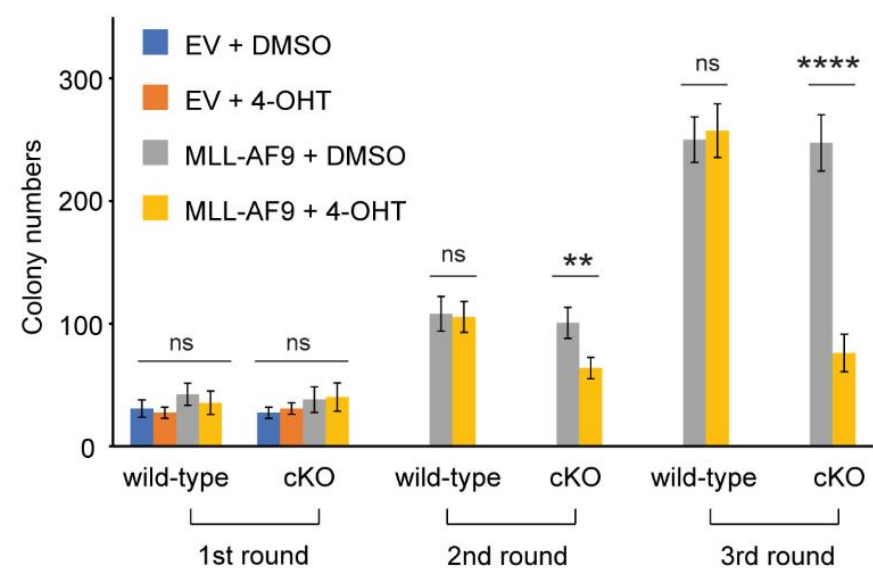

b

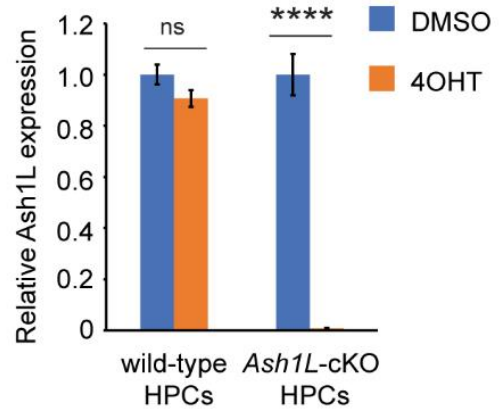

d
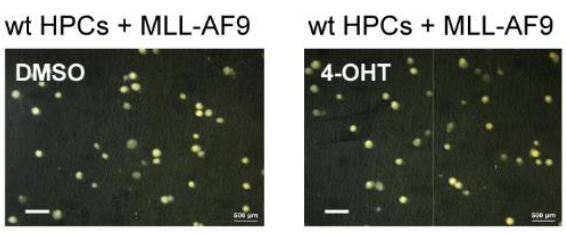

cKO HPCs+ MLL-AF9
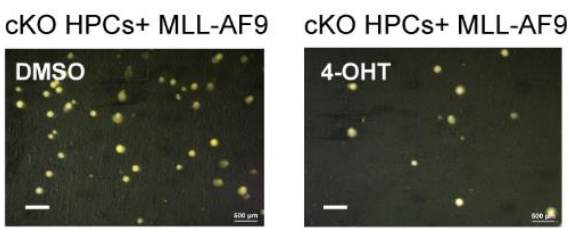

Figure 1. ASH1L is required for the initiation of MLL-AF9-induced leukemic transformation. (a) Schematic experimental procedure. (b) qRT-PCR analysis showing the AshlL expression levels in wild-type and Ash $1 L$-cKO cells after treated with 4-OHT or DMSO. The results were normalized against levels of Gapdh and the expression level in DMSO-treated cells was arbitrarily set to 1 . The error bars represent mean \pm SEM, $\mathrm{n}=3$ per group. ${ }^{*} P<0.05$; $* * P<0.01$; $* * * P<0.001$, ns, not significant. (c) Methylcellulose replating assays showing the colony numbers for each round of plating. The error bars represent mean \pm SEM, $\mathrm{n}=3$ per group. $* * P<0.01 ; * * * *<0.0001$, ns, not significant. (d) Photos showing the representative colony formation on methylcellulose plates for each group. Bar $=0.5 \mathrm{~mm}$. 
Aljazi et al.

a

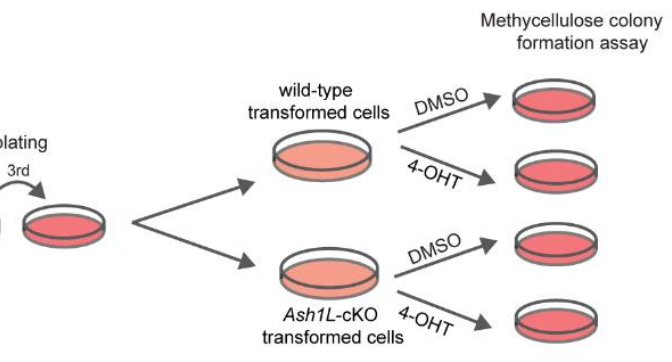

b

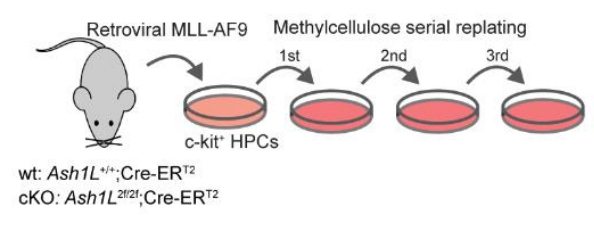

C
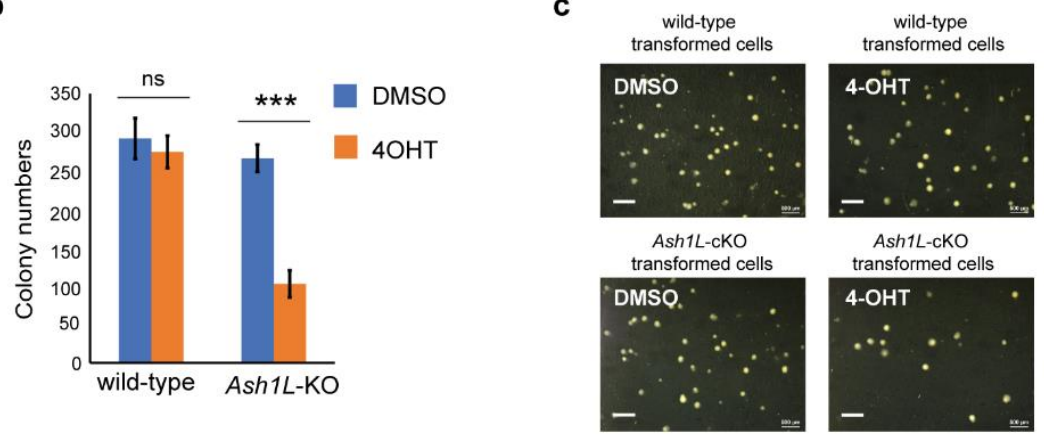

d

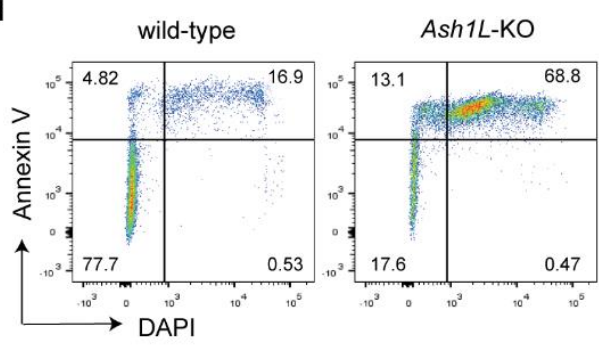

e

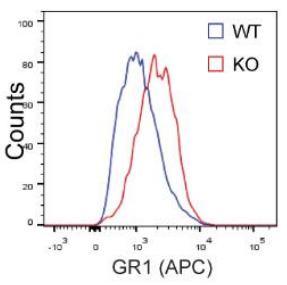

f

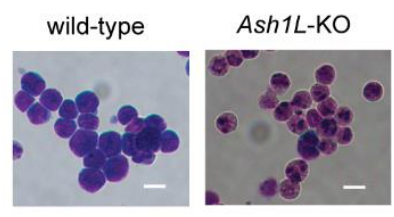

Figure 2. ASH1L is required for the maintenance of MLL-AF9-induced leukemic cells in vitro. (a) Schematic experimental procedure. (b) Methylcellulose colony formation assays showing the colony numbers. The error bars represent mean \pm SEM, $\mathrm{n}=3$ per group. $* * * P<0.001$; ns, not significant. (c) Photos showing the representative colony formation on methylcellulose plates for each group. Bar $=0.5 \mathrm{~mm}$. (d) Representative FACS results showing the Annexin V+ and DAPI+ populations of wild-type and AshlL-KO MLL-AF9-transformed cells. (e) Representative FACS results showing the GR1 and CD11b expression of wild-type and Ash1L-KO MLL-AF9-transformed cells. (f) Photos showing the Wright-Giemsa staining of wild-type and AshlL-KO MLL-AF9-transformed cells. Bar $=10 \mu \mathrm{m}$. 
Aljazi et al.

a

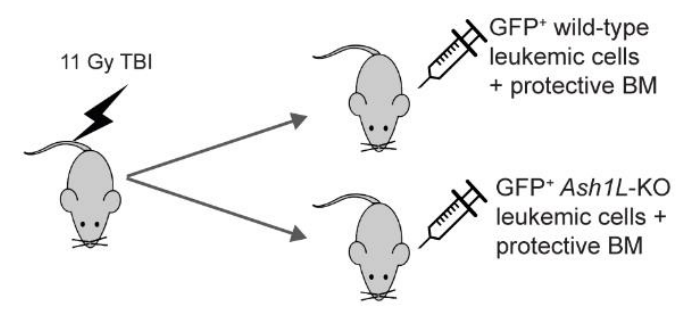

C

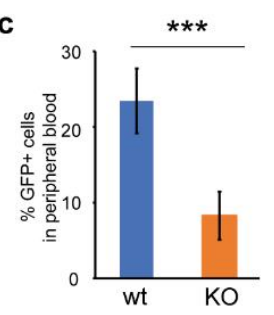

b

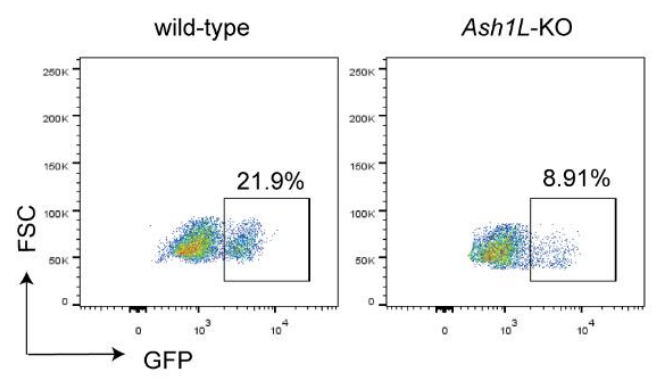

e

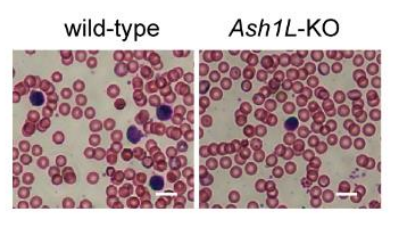

f

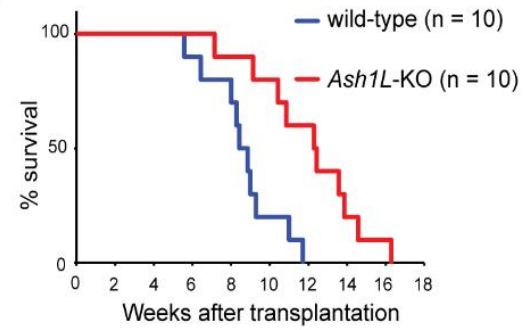

Figure 3. ASH1L is required for the MLL-AF9-induced leukemia development in vivo. (a) Schematic experimental procedure. (b) Representative FACS analysis showing the GFP+ leukemic cell populations in the peripheral blood of mice transplanted with wild-type or Ash1L-KO MLL-AF9transformed cells. The error bars represent mean \pm SEM, $\mathrm{n}=3$ per group. $* * * P<0.001$; ns, not significant. (c) Quantitative results showing the percentage of GFP+ leukemic cell populations in the peripheral blood of mice transplanted with wild-type or $A \operatorname{sh} 1 L$-KO MLL-AF9-transformed cells. The error bars represent mean \pm SEM, $\mathrm{n}=5$ per group. $* * P<0.01$. (d) Photos showing the leukemic cells in the peripheral blood smear of mice transplanted with wild-type or Ash1L-KO MLL-AF9-transformed cells. (e) Photos showing the representative spleen size from the normal control mice (Normal ctrl.), mice transplanted with wild-type (WT) or Ash1L-KO (KO) MLL-AF9-transformed cells. (f) The survival curve of mice transplanted with wild-type or AshlL-KO MLL-AF9-transformed cells. $\mathrm{n}=15$ mice per group. 
Aljazi et al.

a

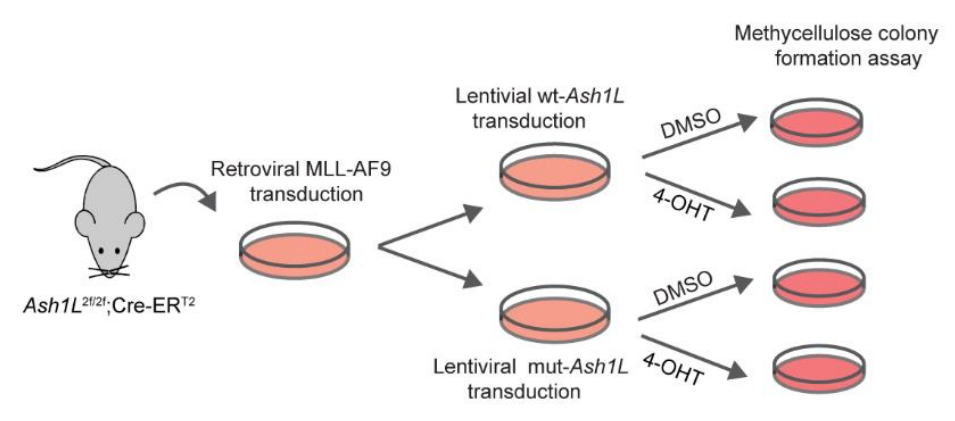

C

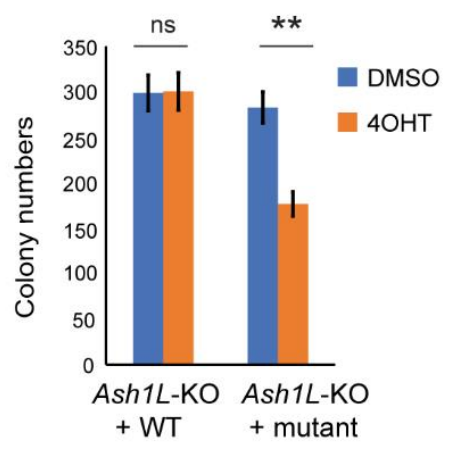

e

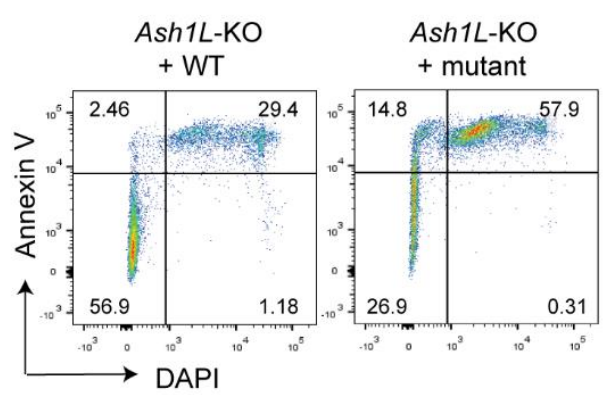

d

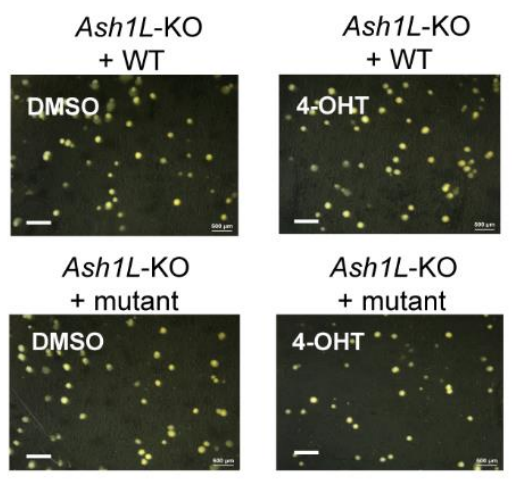

f

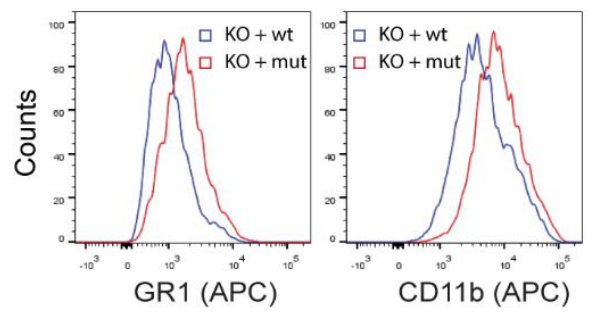

Figure 4. The enzymatic activity of ASH1L is required for its function in promoting MLLAF9-induced leukemic transformation. (a) Schematic experimental procedure. (b) WB analysis showing the ectopic expression of wild-type and mutant ASH1L. (c) Methylcellulose colony formation assays showing the colony numbers. The error bars represent mean $\pm \mathrm{SEM}, \mathrm{n}=3$ per group. $* * P<0.01$; $\mathrm{ns}$, not significant. (d) Photos showing the representative colony formation on methylcellulose plates for each group. Bar $=0.5 \mathrm{~mm}$. (e) Representative FACS results showing the Annexin V+ and DAPI+ populations of Ash1L-KO cells rescued with wild-type and mutant ASH1L. (f) Representative FACS results showing the GR1 and CD11b expression of Ash1L-KO cells rescued with wild-type and mutant ASH1L. 
Aljazi et al.

a

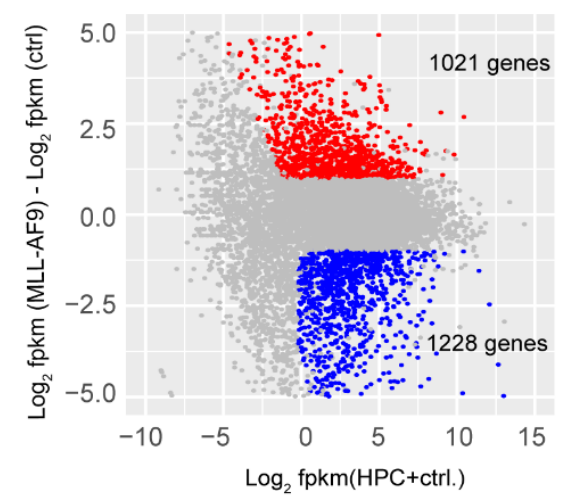

b

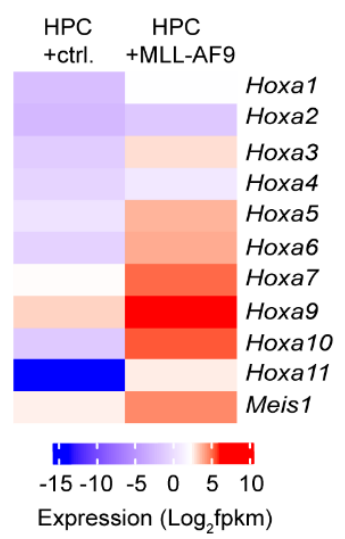

c

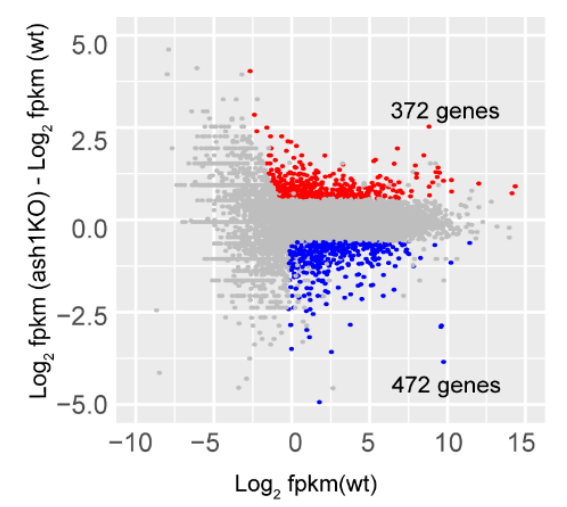

d

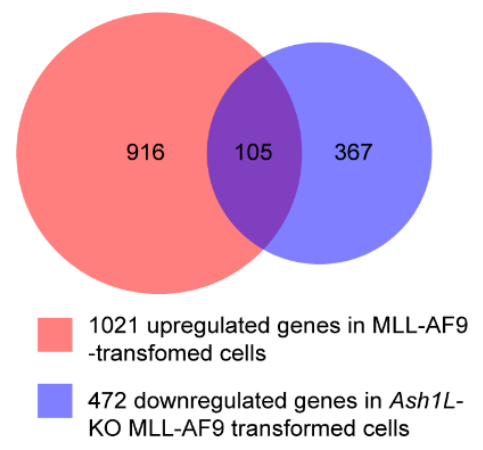

e

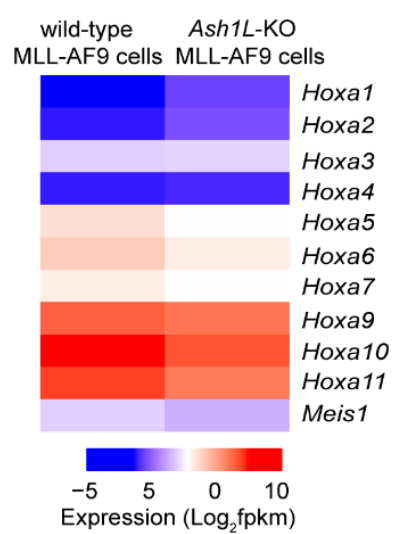

Figure 5. ASH1L facilitates the MLL-AF9-induced leukemogenic gene expression. (a) Plot showing 1021 up- and 1228 down-regulated genes in the MLL-AF9-transformed cells compared to the normal HPCs. (b) Heatmap showing the upregulation of Hoxa gene cluster and MeisI in the MLL-AF9transformed cells compared to normal HPCs. (c) Plot showing 372 up- and 472 down-regulated genes in the Ash1L-KO MLL-AF9-transformed cells compared to the wild-type MLL-AF9-transformed cells. (d) Venn diagram showing the 105 genes upregulated in the MLL-AF9-transformed cells and downregulated in the Ash1L-KO cells. (e) Heatmap showing the Hoxa gene cluster and MeisI downregulated in the AshlL-KO cells compared to the wild-type MLL-AF9-transformed cells. 
Aljazi et al.

a

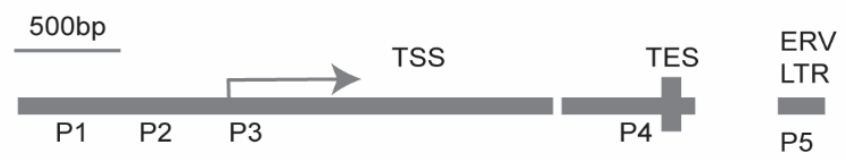

b

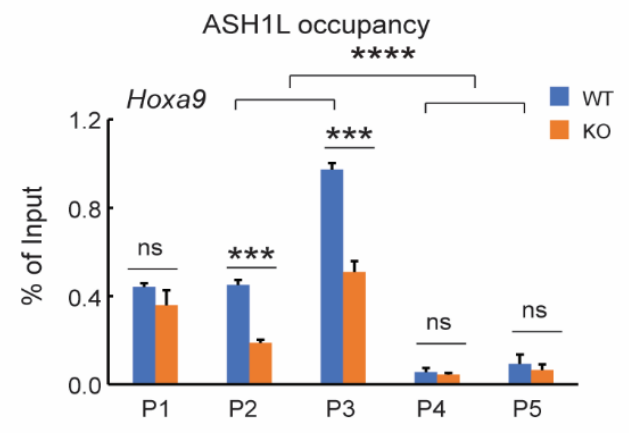

d

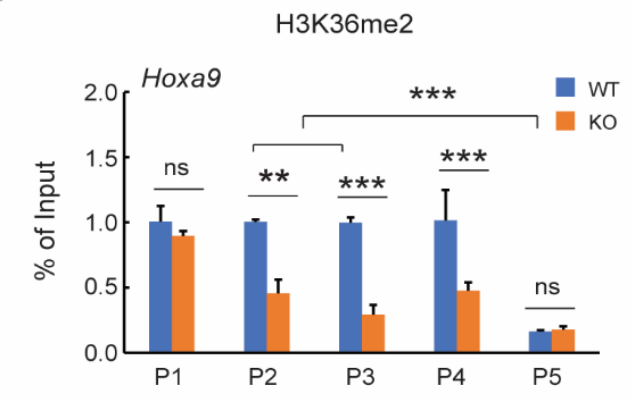

f

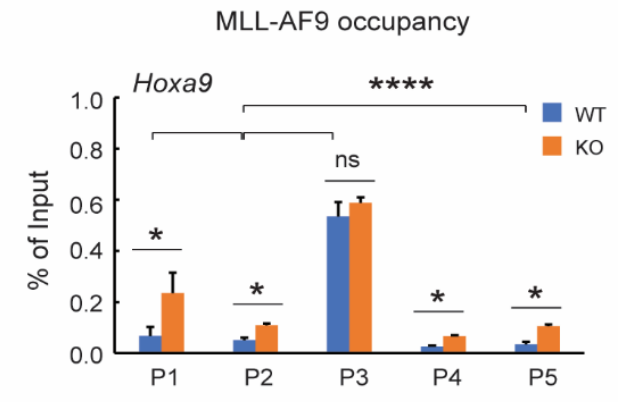

C

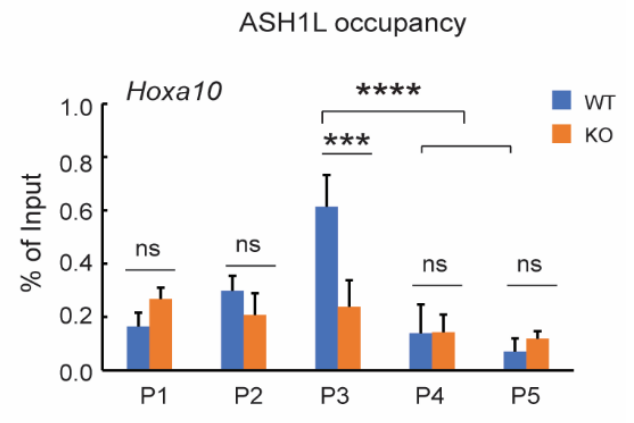

e

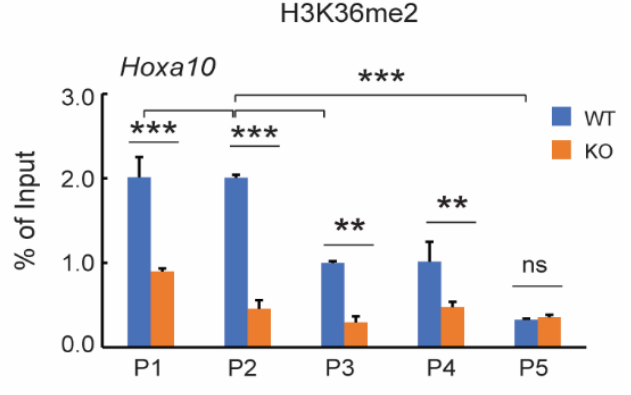

g

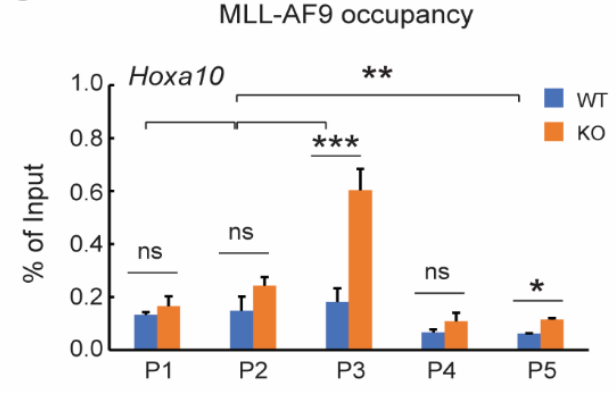

Figure 6. ASH1L binds and mediates histone H3K36me2 modification at Hoxa9 and Hoxa10 gene promoters. (a) Plot showing the locations of ChIP-qPCR amplicons at the Hoxa9 and Hoxal0 gene loci and LTR of endogenous retrovirus. (b-c) ChIP-qPCR analysis showing the ASH1L occupancy at Hoxa9 and HoxalO gene loci in the wild-type and Ash1L-KO MLL-AF9-transformed cells. (d-e) ChIP-qPCR analysis showing the histone $\mathrm{H} 3 \mathrm{~K} 36 \mathrm{me} 2$ at Hoxa9 and HoxalO gene loci in the wild-type and AshlL-KO MLL-AF9-transformed cells. (f-g) ChIP-qPCR analysis showing the MLL-AF9 occupancy at Hoxa9 and Hoxa10 gene loci in the wild-type and Ash1L-KO MLL-AF9-transformed cells. Note: for Figs. b-e, the error bars represent mean $\pm \mathrm{SEM}, \mathrm{n}=3$ biological replicates. $* P<0.05, * * P<0.01$, *** $P<0.001$; **** $P<$ $0.0001 ;$ ns, not significant. 